\title{
Multi-length Scale Cryogenic Sample Preparation to Electron Microscopy of Battery Materials
}

\author{
Cecile Bonifacio, Pawel Nowakowski, Mary Ray and Paul Fischione
}

E.A. Fischione Instruments, Inc., Export, Pennsylvania, United States

Transmission electron microscopy (TEM) is a critical analytical technique for battery research and development. Cryo-electron microscopy (cryo-EM) has been adapted for Li battery research, which allows for the preservation of the battery materials' native state during imaging at the atomic scale. Among the battery components, the anode material, e.g., Li metal, is known to be highly reactive - the material is very sensitive to air and electron beam [1,2]. For this reason, sample preparation and experimentation are challenging because it is necessary to transfer samples between milling systems and microscopes while simultaneously maintaining the material's integrity. A critical requirement in developing these materials for tangible applications is the ability to characterize the materials in a pristine state, without environmental modifications or contamination. Here, we show robust environments that protect the battery material during the sample preparation phase (bulk to focused ion beam [FIB] preparation) and through the multi-length scale electron microscopy characterization phase. At the micrometer scale, scanning electron microscopy (SEM) characterization will show the morphology of the solid electrolyte; at the sub-ångström scale, TEM will provide interfacial chemistry and morphology. High quality, Li ion battery TEM specimens that are free from amorphous and Ga damage will be prepared and imaged under cryo-EM conditions.

Figure 1 shows the controlled environment components for SEM and TEM sample preparation and microscopy characterization. A bulk sample (Figure 1a) was prepared using $\mathrm{Ar}^{+}$broad ion beam (BIB) milling to remove surface oxides and surface damage at cryogenic temperature. A vacuum transfer capsule protected the sample post-BIB milling (Figure 1b). A glove box with a positive-pressure environment was used to transfer the bulk sample from the vacuum transfer capsule to a FIB transfer system (Figure 1c), and thereafter, to a FIB system for morphology and elemental characterization and subsequent TEM specimen preparation. A TEM specimen was prepared in a FIB with a cryo stage at $-170^{\circ} \mathrm{C}$ (Figure 1d-e) using standard lift-out methods and polishing steps (30 and $5 \mathrm{kV}$ ). Subsequently, the TEM specimen was loaded onto a vacuum transfer TEM holder (Figure 1d) that was then moved to a concentrated $\mathrm{Ar}^{+}$beam milling system. $\mathrm{Ar}^{+}$milling was performed by rastering the beam within a defined area of the TEM specimen at decreasing milling energies. The TEM specimen was then loaded on a cryo TEM holder (Figure 1f) inside the glove box; a glove bag with inert gas protected the TEM specimen during insertion of the cryo holder into the TEM. The cryo holder was cooled in the TEM.

Imaging and analysis by electron backscatter diffraction in Figure 2 after cryogenic BIB of Li metal and solid-state electrolyte Li battery show absence of surface oxide, surface damage, and contamination. Energy dispersive X-ray spectroscopy analysis, cryo TEM imaging, and experiments showing removal of FIB-induced damage on TEM specimens are underway. 


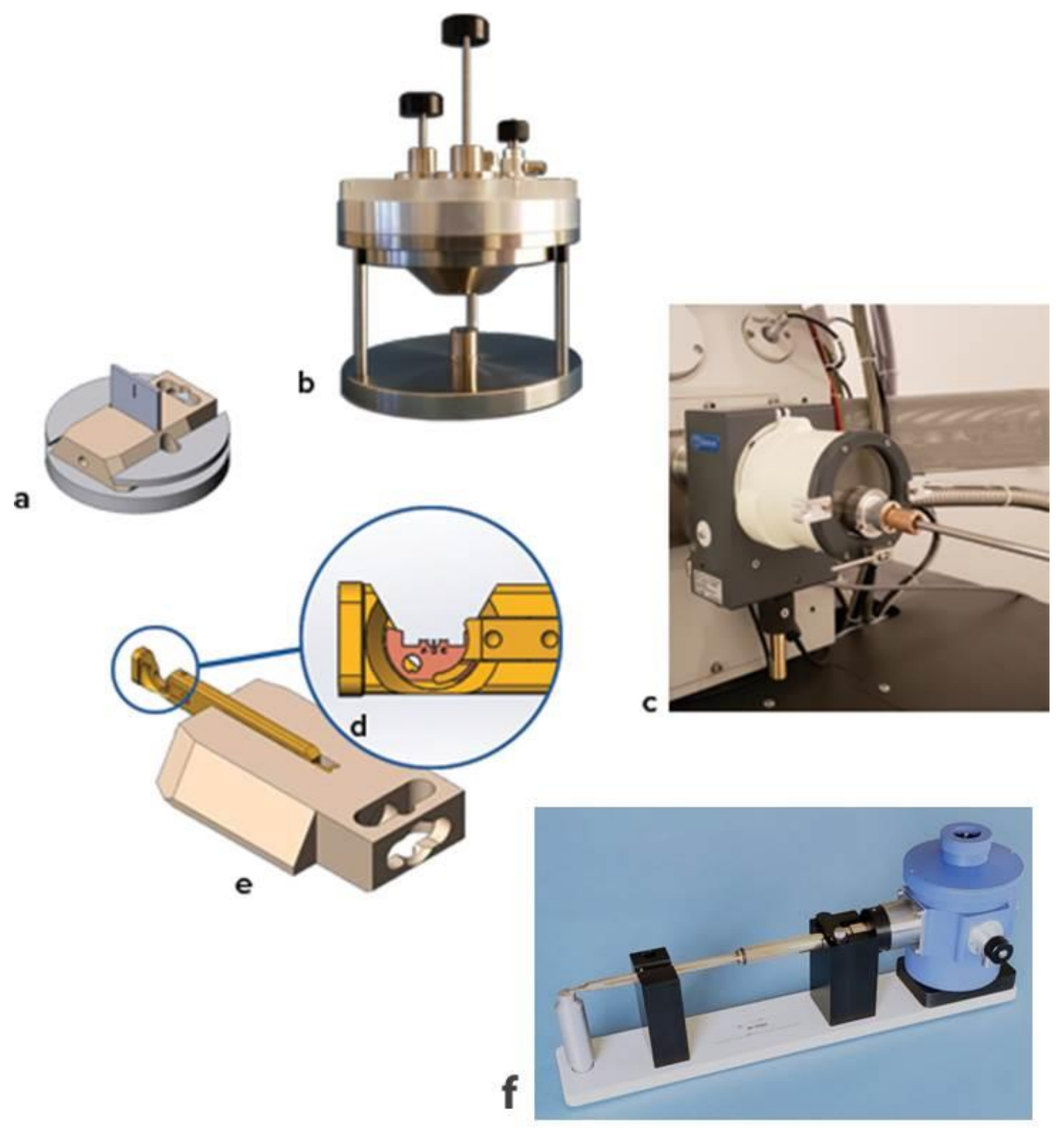

Figure 1. Controlled environments transfer components: (a) scanning electron microscopy (SEM) shuttle for broad ion beam (BIB) milling; (b) vacuum transfer capsule for transfer of BIB-milled sample to glove box; (c) focused ion beam (FIB) transfer system mounted on FIB; (d) transmission electron microscopy (TEM) half-grid mounted on vacuum transfer specimen holder TEM cartridge; (e) TEM cartridge mounted on TEM shuttle and (f) cryo TEM holder. 

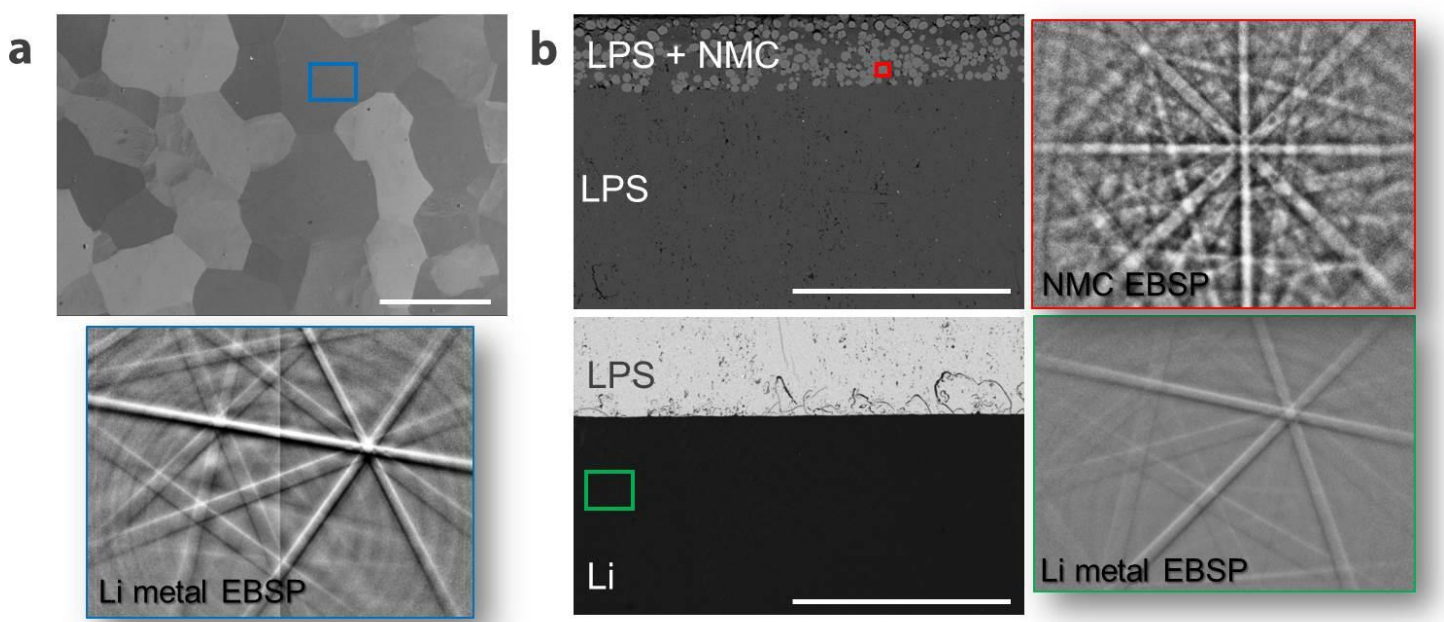

Figure 2. Scanning electron microscopy and electron backscattered pattern images of: a planar Li metal sample (a) and an uncycled solid-state electrolyte Li metal battery cross-section specimen comprising LPS + NMC (Li (NixMnyCo1-x-y)O2) cathode and LPS ((Li3PS4) solid electrolyte and Li metal anode (b) following broad ion beam milling. Scale bar is equal to $200 \mu \mathrm{m}$.

References

[1] X Wang, Y Li and YS Meng, Joule 2 (2018), p. 2225.

[2] F Lin, IM Markus, MM Doeff and HL Xin, Scientific Reports 4 (2015), p. 5694. 